\title{
Natural History of Christianson Syndrome
}

\author{
Richard J. Schroer ${ }^{1,{ }^{*},}$ Kenton R. Holden ${ }^{1,2}$, Patrick S. Tarpey ${ }^{3}$, Maria Giselle Matheus ${ }^{2}$, \\ David A. Griesemer ${ }^{2}$, Michael J. Friez ${ }^{1}$, Jane Zheng Fan ${ }^{4}$, Richard J. Simensen ${ }^{1}$, Petter \\ Strømme $^{5}$, Roger E. Stevenson ${ }^{1}$, Michael R. Stratton ${ }^{3}$, and Charles E. Schwartz ${ }^{1}$ \\ ${ }^{1}$ Greenwood Genetic Center, Greenwood, South Carolina \\ ${ }^{2}$ Medical University of South Carolina, Charleston, South Carolina \\ ${ }^{3}$ Wellcome Trust Sanger Institute, Hinxton, Cambridge, UK \\ ${ }^{4}$ University of North Carolina, Chapel Hill, North Carolina \\ ${ }^{5}$ Faculty of Medicine, University of Oslo, Oslo, Norway
}

\begin{abstract}
Christianson syndrome is an X-linked mental retardation syndrome characterized by microcephaly, impaired ocular movement, severe global developmental delay, hypotonia which progresses to spasticity, and early onset seizures of variable types. Gilfillan et al. [2008] reported mutations in SLC9A6, the gene encoding the sodium/hydrogen exchanger NHE6, in the family first reported and in three others. They also noted the clinical similarities to Angelman syndrome and found cerebellar atrophy on MRI and elevated glutamate/glutamine in the basal ganglia on MRS. Here we report on nonsense mutations in two additional families. The natural history is detailed in childhood and adult life, the similarities to Angelman syndrome confirmed, and the MRI/MRS findings documented in three affected boys.
\end{abstract}

\section{Keywords}

X-linked; intellectual disability; SLC9A6; sodium/hydrogen exchanger; Christianson syndrome

\section{INTRODUCTION}

In 1999, Christianson et al. described an X-linked mental retardation syndrome with severe to profound neurodevelopmental delays including mutism, generalized tonic-clonic epilepsy with electroencephalographic (EEG) abnormalities, truncal/gait ataxia, ophthalmoplegia (convergent strabismus, impaired lateral ocular movement), emaciation, microcephaly, minor craniofacial anomalies (long narrow face with prominent nose, jaw, and ears), and cerebellar and brain stem atrophy on brain magnetic resonance imaging (MRI) and on postmortem examination [Christianson et al., 1999; OMIM 2008] The gene in this Afrikaner family was located between markers DXS424 (Xq24) and DXS548 (Xq27.3).

Gilfillan et al. [2008] identified mutations in SLC9A6, a gene encoding the sodium/ hydrogen exchanger NHE6, in the Afrikaner family and families from Norway, Sweden, and Great Britain. Similarities to Angelman syndrome were noted, including severe to profound mental retardation, mutism, seizures, ataxia, flexed arms, hyperkinesis, happy disposition

"Correspondence to: Richard J. Schroer, M.D., Greenwood Genetic Center, Florence Office, McLeod Medical Park West, 305 East Cheves Street, Suite 370, Florence, SC 29506. schroer@ggc.org. 
with frequent smiling, and unprovoked or easily provoked laughter, open mouth and drooling, swallowing difficulties, and gastroesophageal reflux. Ocular squint was also noted in these families. Progressive cerebellar atrophy on brain MRI and increased glutamate/ glutamine peaks were found in the basal ganglia of some affected males on brain MRS. The mutations identified in the $S L C 9 A 6$ gene were different in the four families.

Herein we report on two families with Christianson syndrome who also presented with an Angelman-like phenotype. Members of one of these families have been followed for over 20 years and provide additional information on the natural history, brain MRI and MRS abnormalities, and the approach to the diagnosis of Christianson syndrome.

\section{CLINICAL REPORTS}

\section{Family 1 (K8310)}

Six males in two generations of this family have profound mental retardation (Figs. 1 and 2). Five affected males, including one subsequently deceased, were examined, and medical records and the autopsy report on the other deceased male were available. Clinical findings are described below and summarized in Table I.

III-9 was born at term with a weight of $3.4 \mathrm{~kg}$ ( 35 th centile), length $53.3 \mathrm{~cm}$ (90th centile), and head circumference (OFC) of $36.8 \mathrm{~cm}$ (80th centile). He was hypotonic and developed tonic-clonic seizures at age 23 months. He never developed speech, but could sit alone, crawl, and walk with assistance. He lost those abilities after age 10 years. At 10 years he was having three to four generalized tonic-clonic seizures a week on phenobarbital. At 26 years, he was hypotonic, could pull-up and stand, but not walk, and was having 0-2 seizures per year on phenobarbital. At 35 years (Fig. 2) he was on phenobarbital and had not had a tonic-clonic seizure in about 10 years. Vision and hearing were normal. His weight was 52 $\mathrm{kg}(<3 \mathrm{rd}$ centile), height $168 \mathrm{~cm}$ (10th centile), and OFC $56 \mathrm{~cm}$ (35th centile). He had an upsweep of his frontal hair, open mouth, drooling, prominent chin, low normal muscle tone, no vertical eye movements, normal lateral and medial ocular movements, pill-rolling movements of his left fingers, 2+ deep tendon reflexes in the upper limbs, 3+ at the knees, $1+$ at the ankles, tight heel cords, and bilateral plantar extensor responses without clonus. He smiled readily and frequently, and repeatedly held up and looked at his hands.

III-10 is presumed to have been affected but did not have mutational analysis. He was born at term with a weight of $3.3 \mathrm{~kg}$ (30th centile), length of $53.3 \mathrm{~cm}$ (90th centile), and OFC of $33 \mathrm{~cm}$ (5th centile). He never learned to speak or walk, developed epilepsy between age 1/1/2 and 2 years, and died at age 4 years. Autopsy revealed microcephaly (OFC $44 \mathrm{~cm} \ll 3 \mathrm{rd}$ centile), short stature (length $82.6 \mathrm{~cm} \ll 3 \mathrm{rd}$ centile), and general cachexia (weight $3.7 \mathrm{~kg}$ $\ll 3$ rd centile). The brain weighed $870 \mathrm{~g}$ and had enlarged lateral ventricles $(6 \mathrm{~cm} \times 10 \mathrm{~cm} \times$ $15 \mathrm{~cm})$ and a dilated fourth ventricle $(2 \mathrm{~cm} \times 1 \mathrm{~cm})$. Histology of sections of the brain was unremarkable. The left testis was undescended, musculature and subcutaneous tissue were virtually absent, but the remainder of the autopsy findings were normal.

III-12 was born at term with a weight of $3.4 \mathrm{~kg}$ ( 35 th centile), length $53.3 \mathrm{~cm}$ (90th centile), and head circumference $33 \mathrm{~cm}$ (5th centile). He had congenital hypotonia. He learned to crawl, sit up, and pull-up but never developed speech, independent ambulation, or toilet control. He had the onset of generalized tonic-clonic seizures at age 7 months. The seizures were poorly responsive to phenobarbital and phenytoin. He had feeding problems from birth, and similar to the other affected males in the family, he did not chew food well and was fed pureed or chopped food. At age 4 years his OFC was $45.2 \mathrm{~cm}$ ( $\ll 3 \mathrm{rd}$ centile). At 28 years (Fig. 2), he was being treated with phenytoin and valproic acid and had had two generalized tonic «clonic seizures in the last year. His OFC was $48.5 \mathrm{~cm}(\ll 3 \mathrm{rd}$ centile). In 
addition to microcephaly, he had generalized asthenia, a thin gaunt face, upswept frontal hair, sunken eyes, high and narrow nasal bridge, short philtrum, low nasal septum, open mouth with everted lower lip, and marked scoliosis. He had normal strength for his decreased muscle bulk, increased tone with no abnormal movements or contractures, deep tendon reflexes in the upper limbs of $1+$ to $2+$ and in the lower limbs of zero to $1+$, and plantar flexor responses. He appeared to have impaired vertical eye movement but had normal medial and lateral movements. He frequently placed his palms in front of his eyes, clapped, smiled, or laughed. He was non-verbal and non-ambulatory. At age 29 years he died suddenly apparently from an epileptic seizure.

IV-1 was born at 38 weeks of gestation with a weight of $2.5 \mathrm{~kg}$ (10th centile), length 49.5 $\mathrm{cm}$ (55th centile), and OFC $29.8 \mathrm{~cm}$ ( 3 rd centile). At 4.5 months, he was hypotonic and unsteady when supported. Before age 2 years he had the onset of generalized tonic-clonic seizures that were treated with phenobarbital. At age 2 years, he was non-verbal, but was pulling up, crawling and hand feeding, but his movements were slow and deliberate. He developed the ability to walk with assistance. He lost all these motor abilities with worsening of generalized tonic-clonic and atonic seizures at about age 10 years. He had been hypotonic with all his limb deep tendon reflexes $1+$ to $2+$ and with plantar flexor responses. On examinations after 10 years, all his deep tendon reflexes were $3+$ to $4+$ with sustained clonus at the ankles and with plantar extensor responses. He also developed contractures, and at age 13 years he had bilateral hip flexor releases, bilateral hamstring lengthening, and bilateral heel cord releases. By 14 years being treated with phenobarbital, valproate, and levetiracetam, tonic-clonic seizures were noted in his sleep once or twice per week. At age 14 years (Fig. 2), he remained non-verbal and had a propensity to look with his head tilted back, laughed, and giggled at some sounds, including phone rings and bells, and had recurrent hypothermia. He had a hypotonic face, abnormal horizontal eye movements bilaterally (Fig. 3), incomplete extension at his elbows and knees, tight heel cords, right thoracolumbar scoliosis, generalized mild hypotonia, and a tendency to clap spontaneously and hold his palms in front of his face. His weight was $28.6 \mathrm{~kg}$ ( $\ll 3 \mathrm{rd}$ centile), length 134 $\mathrm{cm}(\ll 3 \mathrm{rd}$ centile), and OFC $49.5 \mathrm{~cm}(\ll 3 \mathrm{rd}$ centile).

IV-4 was born after an uncomplicated 38-week gestation weighing $2.4 \mathrm{~kg}$ (5th centile). He had congenital hypotonia. At age 1 year his weight was $8.6 \mathrm{~kg}$ ( 5 th centile), length $75 \mathrm{~cm}$ ( 45 th centile), and head circumference $42 \mathrm{~cm}$ ( $\ll 3 \mathrm{rd}$ centile). He sat at 14 months and walked at 2.5 years. He walked with poor balance and usually kept his head tilted back when walking. He acquired the abilities to hold his bottle and to finger feed. He had the onset of generalized tonic-clonic seizures at 15 months. With the onset of seizures and typical abnormal EEG findings, a diagnosis of Lennox-Gastaut syndrome was proposed. His developmental quotient was 50 and $\mathrm{OFC} 43 \mathrm{~cm}(\ll 3 \mathrm{rd}$ centile) at age 17 months. His adopted family reported he had trouble rotating his eyes vertically. At age 4 years he had surgical correction of bilateral esotropia. He had worsening of his complex partial seizures with secondary generalized tonic-clonic, atypical absence, and atonic seizures even while on multiple seizure medications between age 6 and 7 years. The EEG contained frontal highamplitude 2-3-Hz rhythmic spike-wave activity in addition to other findings consistent with Lennox-Gastaut syndrome. He also developed a right hemiparesis. Although his tone was increased on the right, his deep tendon reflexes and plantar responses were normal. He subsequently lost almost all abilities. His neurodevelopmental quotient was assessed as 20 . At age $6^{11 / 12}$ years (Fig. 2), his weight was $17.7 \mathrm{~kg}$ (3rd centile), length $113 \mathrm{~cm}$ (5th centile), and OFC was $46.5 \mathrm{~cm}(\ll 3 \mathrm{rd}$ centile).

IV-6 was first evaluated at age 9 months. He had rolled over but was not able to sit unsupported. His weight was $7.5 \mathrm{~kg}$ (<3rd centile), length $70.2 \mathrm{~cm}$ (20th centile), and OFC $39.8 \mathrm{~cm}$ ( $\ll 3$ rd centile). Results of immunodeficiency evaluation for recurrent upper 
respiratory infections during early infancy were normal. At 14 months (Fig. 2) he could sit alone and stand with support, but used no words. His weight was $7.73 \mathrm{~kg}(<3 \mathrm{rd}$ centile), length $75.3 \mathrm{~cm}$ (20th centile), and OFC $41.4 \mathrm{~cm}$ ( $\ll 3 \mathrm{rd}$ centile). He had a triangular face with upswept frontal hair and a wide mouth with thin upper lip. He had a happy disposition and was hyperkinetic. He developed tonic-clonic seizures at 16 months. His EEG was normal. The seizures were poorly controlled by carbamazepine and he was switched to levetiracetam. He has been hospitalized twice for uncontrolled seizures. One hospitalization was for convulsive status epilepticus. At about 2 years he developed infrequent atypical absence seizures. He walked independently at 26 months but with unsteadiness and ataxia. He also exhibited impaired horizontal eye movements bilaterally and bilateral nystagmus with visual fixation. At 33 months his estimated developmental quotient was 30 .

II-4, an obligate carrier, had "learning problems" and completed 4 years of school. She has a speech disorder but no hearing impairment. Her adult head circumference was $54.2 \mathrm{~cm}$ (40th centile). Her carrier daughter, III-11, also had "difficulty in school," particularly in math and reading, and she withdrew from school in the ninth grade. Her non-carrier daughter, III-16, had average performance in school and withdrew in the 11th grade. The results of cognitive testing with the Stanford-Binet on these two carriers and one noncarrier are given in Table II.

III-11 had 7 children including three affected sons (IV-1, IV-4, and IV-6) and a non-carrier daughter (IV-3) who were examined.

IV-7, a 2-year-old carrier, has "mild neurodevelopmental delays, hyperkinesis, and mild truncal ataxia."

III-7 and III-8, two unaffected sons of II-4, both completed nine grades of school and have head circumferences of $56.5 \mathrm{~cm}$ (60th centile) and $58 \mathrm{~cm}$ (95th centile), respectively.

IV-3, an 81/2-year-old non-carrier daughter of III-11, has learning problems in reading and math and receives speech therapy. She repeated kindergarten and completed the first grade. She also has behavior problems including aggressiveness. She has been diagnosed with attention deficit-hyperactivity disorder and oppositional-defiant disorder. She had a normal EEG and a normal brain MRI when evaluated for absence seizure-like episodes. A glucose tolerance test revealed "borderline" hypoglycemia. Her OFC was $51.5 \mathrm{~cm}$ (50th centile) with upswept frontal hair.

IV-5, a 5-year-old non-carrier, has "normal neurodevelopment."

\section{Family 2 (K9524)}

The propositus was noticed to have decreased intrauterine movements but was born at term by vaginal-vertex delivery with normal Apgar scores and weight $3.3 \mathrm{~kg}$ (30th centile), length $50.8 \mathrm{~cm}$ (60th centile), and OFC $33 \mathrm{~cm}$ (5th centile). He had hypoactivity, poor feeding, and infrequent crying from birth. Seizures began at 6 months. He was diagnosed and treated for complex partial epilepsy at 10 months. He was delayed in motor abilities and in social interactions from birth. At 14 months his Bayley Scales of Infant Development was 43 . He developed but subsequently lost the abilities to crawl, pull up, and cruise with his first cluster of tonic-clonic seizures at 2 years. He had gastroesophageal reflux, poor feeding, poor chewing, and poor control of secretions. He had a G-tube placement and Nissen fundoplication at 2 years and thereafter has been exclusively G-tube fed. He had surgery for bilateral esotropia at 16 months. At 8 months his OFC was $40.5 \mathrm{~cm}$ ( $\ll 3 \mathrm{rd}$ centile). At age 6 years (Fig. 2) his weight was $20.6 \mathrm{~kg}$ (40th centile), and length $105.4 \mathrm{~cm}$ (3rd centile), and 
OFC was $46 \mathrm{~cm}$ ( $\ll 3 \mathrm{rd}$ centile). In addition to hypotonia, he had areflexia and plantar flexor responses.

An electroretinogram (ERG) at 16 months was normal. Brain MRI at about 3 years reportedly showed mild cerebellar volume loss involving the vermis and the inferior aspects of the cerebellum but was not available for review. Video-EEG recorded three generalized seizures with bifrontal dominance of multifocal spikes with burst activity and interictal discharges with a predominant alpha rhythm throughout the interictal discharges. His generalized tonic-clonic seizures were difficult to control with anticonvulsants, were not controlled by a liquid ketogenic diet, and were best controlled with a vagal nerve stimulator (VNS), oxcarbazepine, and clonazepam. His generalized tonic-clonic seizures decreased markedly with these therapies, but atypical absence seizures became more frequent.

He had extensive studies including $U B E 3 A$ and $M E C P 2$ mutational analysis, CSF neurotransmitter analysis, and muscle mitochondrial enzymology before finding his SLC9A6 mutation. CSF glycine, asparagine, and glutamic acid were elevated. The remainder of CSF amino acids, including glutamine, and neurotransmitters were normal.

\section{METHODS AND RESULTS}

\section{Brain MRI and MR Spectroscopy}

Brain magnetic resonance imaging (MRI) was performed on three of the affected brothers from Family 1 (IV-1, IV-4, and IV-6). Single voxel magnetic resonance spectroscopy (MRS) was completed on two of the affected brothers (IV-1, IV-4). The brain MRI examination included isotropic gradient echo T1-weighted, acquired in sagittal with three plane reconstructions, transverse T2-weighted, transverse and coronal fluid attenuation inversion recovery (FLAIR), transverse diffusion weighted and coronal inversion recovery T1-weighted images. MRS assessment included single voxel short and long echo time (2530 and $135 \mathrm{~ms}$, respectively). Volumes of interest for the MRS were placed in the left basal ganglia on subject IV-1, as well as in bilateral basal ganglia and bilateral parietal white matter on subject IV-4.

The imaging findings in the older brothers (IV-1 and IV-4) demonstrated focal atrophy and/ or hypoplasia of the inferior cerebellar vermis with a subtle associated high signal seen in the inferior aspect of the cerebellar hemispheres on coronal FLAIR sequences in both patients. These abnormal FLAIR findings may indicate some degree of gliosis. The youngest brother's (IV-6) MRI at age 15 months exhibited a prominent cerebello-medullary cistern which may reflect mild volume loss of the inferior vermis, and mild increased FLAIR signal in the posterior periventricular white matter. The cerebellar atrophy and/or hypoplasia, although present in a similar location, was more conspicuous in the oldest subject (IV-I) (Fig. 4). Subject IV-1 at age 14 years also showed a mild volume loss of the periventricular white matter with prominent lateral ventricles, especially in the occipital horns (Fig. 4). The hippocampi of subjects IV-1 and IV-4 were reviewed on multiple coronal, axial, and sagittal sequences. Mild bilateral hippocampal atrophy was present in subject IV-1 accompanied by subtle bilateral associated high signal in both hippocampi on the coronal FLAIR, which may also indicate some degree of gliosis (Fig. 4). The hippocampi of subjects IV-4 and IV-6 were within normal limits (Fig. 5).

Brain MRS in IV-1 and IV-4 demonstrated to some degree a similar abnormal spectrum in all five assessed locations. These findings consisted of mildly decreased levels of metabolites, especially NAA/creatinine ratios, and a prominent glutamine/glutamate shoulder evident on short echo time sequence (Fig. 6). 


\section{Molecular Genetics}

Primers were designed to individually amplify exons $1-16$ of the $S L C 9 A 6$ gene. The amplicons were sequenced with a standard Big Dye protocol from Applied Biosystems and run on an ABI3730 capillary instrument (Applied Biosystems, Foster City, CA). Generated sequences were aligned with sequence data from normal controls as well as SLC9A6 text sequence from Ensembl.

In Family 1 (K8310), the affected males and the obligate carrier females were found to have a c.1498C $\rightarrow$ T alteration in $S L C 9 A 6$ (Table III). This alteration produces a stop codon at amino acid 468 (R468X). This is the same mutation as reported in a Swedish family by Gilfillan et al. [2008]. In Family 2, the propositus has c.1219C $\rightarrow$ T mutation in SLC9A6, resulting in a stop codon replacing a glutamine at 407 (p.Q407X). The mutation is de novo since his mother has two normal copies of the SLC9A6 gene.

\section{DISCUSSION}

Christianson syndrome usually presents in infancy with acquired microcephaly, undergrowth and global neurodevelopment delays. Affected boys exhibit limited fine and gross motor development with ataxia, absent or very limited speech development, intractable epilepsy, and eventually regression in previously acquired skills.

Delayed neurodevelopment is evident within the first 6 months of life and is associated with mild-to-moderate generalized hypotonia. The affected males may develop the ability to hold a bottle or to feed with their hands. They make efforts toward ambulation or attain some degree of independent ambulation but with an ataxic gait. There is usually no speech, but if it develops, it is limited to a few words. They do not develop toileting or other self-help skills. They may have poor feeding from birth. Even when older, they require soft foods. They do not chew or swallow well, and exhibit excessive drooling. They have an asthenic habitus. There may not be any particular facial abnormalities, but the gaunt facies give prominence to other facial structures - the ears, the nose, and the jaw. Although there are no definite vision and hearing impairments, impaired ocular movements are frequent. These include esotropia and impaired lateral and/or vertical ocular movements. Female carriers have been previously reported to have learning and behavioral problems [Christianson et al., 1999; Gilfillan et al., 2008]. However, in kindred K8310 both carriers and non-carriers have learning, school, and behavior problems,so these problems may not be specific to female carrier status in this family.

Affected males lose attained motor abilities, usually by the end of the first decade. Some develop hyperreflexia, spasticity, and plantar extensor responses. They are or become completely dependent but remain responsive to people, sights, and noises in the environment. They may have affable or happy dispositions and may be easily provoked to or spontaneously exhibit smiling/laughing. It is unclear if the neurodevelopmental regression is related solely to the progression of the syndrome and/or to the severe uncontrolled seizures with/without accompanying anti-epileptic drug side effects.

Almost all affected males have microcephaly, and it is usually of the acquired type. Head circumferences at birth were in the lower centiles (3rd to 5th centiles) in four affected infants and at the 80th centile in one. The latter had a normal head circumference (35th centile) as an adult but all the other affected males developed microcephaly ( $<3$ rd centile). Three of four patients in the family reported by Christianson et al. [1999] had abnormally small head circumferences. Gilfillan et al. [2008] described deceleration of head growth in the first year of life. 
Affected males commonly develop generalized tonic-clonic seizures within the first 2 years of life. Other seizure types (atypical absence, atonic, complex partial) frequently follow. All the seizure types appear poorly responsive to antiepileptic therapies. In those patients IV-1 and IV-4 in family 1 who have been followed for epilepsy by one of the authors (DG), the seizure semiology and neurodevelopmental course were indistinguishable clinically and electrographically from that of typical Angelman syndrome. For example, the EEG findings in IV-4 were typical of those reported in Angelman syndrome [Laan et al., 1998]. Gilfillan et al. [2008] reported Angelman-like slow frequency $1.5-3 \mathrm{~Hz}$ with high voltage $(300 \mathrm{mV})$ in only one patient but $10-14 \mathrm{~Hz}$ rapid background EEG in others.

Brain MRIs were performed on three affected brothers, IV-1 at 14 years, IV-4 at 7 years, and IV-6 at 15 months (Figs. 4 and 5). The brain MRIs revealed focal atrophy and/or hypoplasia of the inferior cerebellar vermis in both of the older brothers (IV-1 and IV-4) but more marked in the oldest brother (IV-1) (Fig. 5C,D). Brain MRI on the youngest IV-6 revealed no significant cerebellar abnormalities; however, a prominent cerebello-medullary cistern was evident, which may reflect a mild volume loss in the inferior aspect of the cerebellar vermis. Prominent lateral ventricles, especially the occipital horns, with mild periventricular white matter volume loss and hippocampal atrophy/hypoplasia were noted in IV-1 but not in IV-4 (Fig. 5A-D). It is unclear if the ventricular prominence is congenital or related to atrophy. On brain MRS, there were bilaterally increased glutamate/glutamine shoulders (peaks) in the basal ganglia in IV-1 and IV-4 and in the parietal white matter in IV-1 (Fig. 6). The cerebellar atrophy has been previously described as progressive [Gilfillan et al., 2008]. The differences in the neuroimaging in the older and younger brothers in this report support atrophy rather than hypoplasia of the cerebellar vermis, the inferior cerebellar hemispheres, and the hippocampi.

Heterozygous loss of function mutations of $C A S K$ in four girls and a splice site mutation in one boy caused a similar intellectual disability syndrome with microcephaly and hypoplasia of the cerebellum and brain stem [Najm et al., 2008]. Mutations in both SLC9A6 and CASK produce ocular findings, abducens or other ocular palsies associated with strabismus and nystagmus being most common. The Angelman syndrome-like demeanor noted with SLC9A6 mutations has not been noted with CASK mutations [Anna Hackett, personal communication, 2009]. Piluso et al. [2003, 2009] described a missense mutation of $C A S K$ in 3 males in a family with normal OFC, hypertelorism, frontal hair upsweep, constipation, hypotonia, aggressive behavior, and epilepsy. There was no report of ocular or cerebellar findings or signs of Angelman-like demeanor. A brain CT scan in 1 male was normal. This very different presentation was considered to represent a variant of FG syndrome [Piluso et al., 2009].

The relationship of the seizures, the elevated glutamate/glutamine, and the hippocampalcerebellar atrophy is complex. Glutamate is the primary brain excitatory neurotransmitter and is associated with neurodegeneration and with seizures [Simeone et al., 2004]. Glutamergic excitotoxicity is proposed to be associated with seizures and with progressive cerebellar atrophy [Orsi et al., 1988; Plaitakis et al., 1992; Cavus et al., 2008]. High glutamate may cause hippocampal atrophy or vice versa or there may be a common mechanism for both [Cavus et al., 2008]. Epilepsy may be associated with decreased hippocampal volume and elevated brain glutamate [Cavus et al., 2008]. Increased seizure frequency may contribute to hippocampal damage [Cavus et al., 2008]. In Christianson syndrome there is neurodevelopmental regression which appears to accompany the appearance of atrophy of cerebellar vermis, cerebellar hemispheres, and hippocampi. The clinical and hippocampal-cerebellar deterioration may indicate that Christianson syndrome is a neurodegenerative condition and/or may result from damage from the increasing frequency and severity of intractable seizures. 
The mutations reported to date in $S L C 9 A 6$ have been nonsense or frameshift alterations leading to premature truncation of the SLC9A6 protein. These findings suggest that complete or profound loss of functional SLC9A6 protein is required for the Angelman-like or severe presentation of Christianson syndrome. SLC9A6 mutations that do not lead to a complete loss of functional protein might cause less severe phenotypes. SLC9A6 encodes a sodium/hydrogen exchanger which appears to be localized to the mitochondria and which among other functions regulates $\mathrm{pH}$ and cell volume [Numata et al., 1998]. Mutations in an autosomal member of the sodium/hydrogen exchanger family, SLC9A9, have been associated with epilepsy and autism [Morrow et al., 2008]. Although males with SLC9A6 mutations have not been formally evaluated for autism, they have lack of speech and profound intellectual disability. Missense and null mutations in sodium channel genes cause generalized epilepsy with febrile seizures plus (GEFS+2) and the more severe myoclonic epilepsy of infancy, respectively; and polymorphic variants in sodium channel genes may contribute to autism [Weiss et al., 2003].

SLC9A6 mutations should be considered in the differential diagnosis of males with X-linked mental retardation and a Christianson syndrome phenotype or in younger males with an Angelman syndrome phenotype with otherwise normal molecular genetic studies. Although there are similarities between Christianson syndrome and Angelman syndrome, Christianson syndrome has impaired ocular movements and neurodevelopmental regression. Christianson syndrome also develops decreased cerebellar and hippocampal volumes on brain MRI and has abnormal glutamine/glutamate peaks on brain MRS.

\section{REFERENCES}

Cavus I, Pan JW, Hetherington HP, Abi-Saab W, Zaveri HP, Vives KP, Krystal JH, Spencer SS, Spencer DD. Decreased hippocampal volume on MRI is associated with increased extracellular glutamate in epilepsy patients. Epilepsia. 2008; 49:1358-1366. [PubMed: 18410365]

Christianson AL, Stevenson RE, van der Meyden CH, Pelser J, Theron FW, van Rensburg PL, Chandler M, Schwartz CE. X-linked severe mental retardation, craniofacial dysmorphology, epilepsy, ophthalmoplegia, and cerebellar atrophy in a large South African kindred is localized to Xq24-q27. J Med Genet. 1999; 36:759-766. [PubMed: 10528855]

Gilfillan GD, Selmer KK, Roxrud I, Smith R, Kyllerman M, Eiklid K, Kroken M, Mattingsdal M, Egeland T, Stenmark H, Sjøholm H, Server A, Samuelsson L, Christianson A, Tarpey P, Whibley A, Stratton MR, Futreal PA, Teague J, Edkins S, Gecz J, Turner G, Raymond FL, Schwartz C, Stevenson RE, Undlien DE, Strømme P. SLC9A6 mutations cause X-linked mental retardation, microcephaly, epilepsy, and ataxia, a phenotype mimicking Angelman syndrome. Am J Hum Genet. 2008; 82:1003-1010. [PubMed: 18342287]

Laan LA, Brouwer OF, Begeer CH, Zwinderman AH, van Dijk JG. The diagnostic value of the EEG in Angelman and Rett syndrome at a young age. Electroencephalogr Clin Neurophysiol. 1998; 106:404-408. [PubMed: 9680152]

Morrow EM, Yoo SY, Flavell SW, Kim TK, Lin Y, Hill RS, Mukaddes NM, Balkhy S, Gascon G, Hashmi A, Al-Saad S, Ware J, Joseph RM, Greenblatt R, Gleason D, Ertelt JA, Apse KA, Bodell A, Partlow JN, Barry B, Yao H, Markianos K, Ferland RJ, Greenberg ME, Walsh CA. Identifying autism loci and genes by tracing recent shared ancestry. Science. 2008; 321:218-223. [PubMed: 18621663]

Najm J, Horn D, Wimplinger I, Golden JA, Chizhikov VV, Sudi J, Christian SL, Ullmann R, Kuechler A, Haas CA, Flubacher A, Charnass LR, Uyanik G, Frank U, Klopocki E, Dobyns WB, Jutsche K. Mutations of CASK cause an X-linked brain malformation phenotype with microcephaly and hypoplasia of the brainstem and cerebellum. Nat Genet. 2008; 40:1065-1067. [PubMed: 19165920]

Numata M, Petrecca K, Lake N, Orlowski J. Identification of a mitochondrial Na+/H+ exchanger. J Biol Chem. 1998; 273:6951-6959. [PubMed: 9507001] 
Online Mendelian Inheritance in Man (OMIM). [Accessed August 31, 2008] Mental retardation, Xlinked syndromic, Christianson type. Johns Hopkins University. 2008. Available at: http:// www.ncbi.nlm.nih.gov/entrez/dispomim.cgi?id=300243

Orsi L, Bertolotto A, Brignolio F, Chió A, Meineri P, Mittino D, Mortara P, Schiffer D. Glutamate dehydrogenase (GDH) deficiency in different types of progressive hereditary cerebellar ataxia. Acta Neurol Scand. 1988; 78:394-400. [PubMed: 3218446]

Piluso G, Carella M, D'Avanzo M, Santinelli R, Carrano EM, D'Avanzo A, D'Adamo AP, Gasparini P, Nigro V. Genetic heterogeneity of FG syndrome: A fourth locus (FGS4) maps to Sp11.4-p11.3 in an Italian family. Hum Genet. 2003; 112:124-130. [PubMed: 12522552]

Piluso G, D'Amico F, Saccone V, Bismuto E, Rotundo IL, Di Domenico M, Aurino S, Schwartz CE, Nero G, Nigro V. A missense mutation in CASK causes FG syndrome in an Italian family. Am J Hum Genet. 2009; 84:162-177. [PubMed: 19200522]

Plaitakis A, Flessas P, Natsiou AB, Shashidharan P. Glutamate dehydrogenase deficiency in cerebellar degenerations: Clinical, biochemical and molecular genetic aspects. Can J Neurol Sci. 1992; 20:S109-S116. [PubMed: 8334586]

Simeone TA, Sanchez RM, Rho JM. Molecular biology and ontogeny of glutamate receptors in the mammalian central nervous system. J Child Neurol. 2004; 19:343-360. [PubMed: 15224708]

Weiss LA, Escayg A, Kearney JA, Trudeau M, MacDonald BT, Mori M, Reichert J, Buxbaum JD, Meisler MH. Sodium channels SCN1A, SCN2A and SCN3A in familial autism. Mol Psychiatry. 2003; 8:186-194. [PubMed: 12610651] 


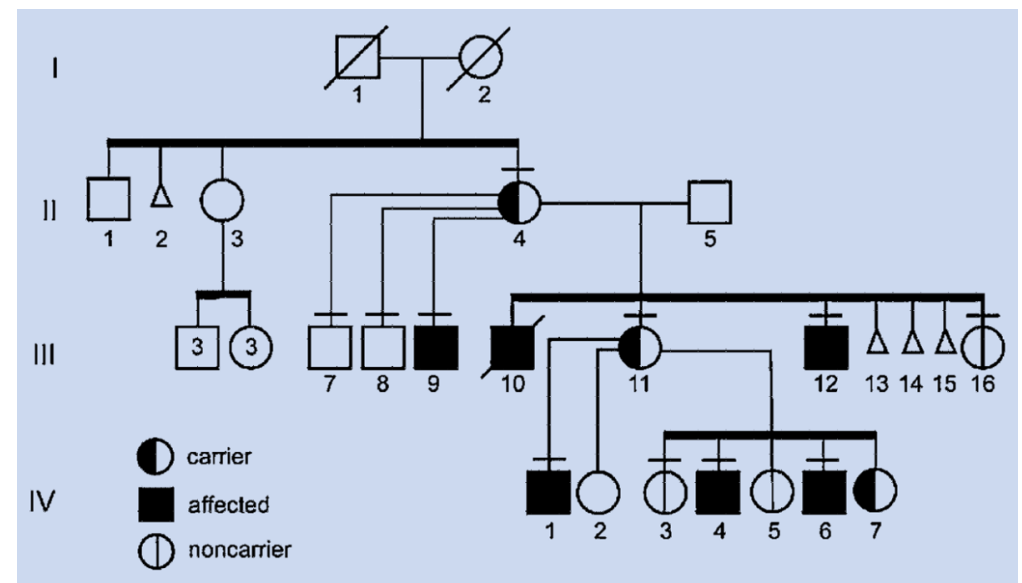

FIG. 1.

Partial pedigree of Family 1 (K8310), showing six affected males in two generations and three carrier females in three generations. 


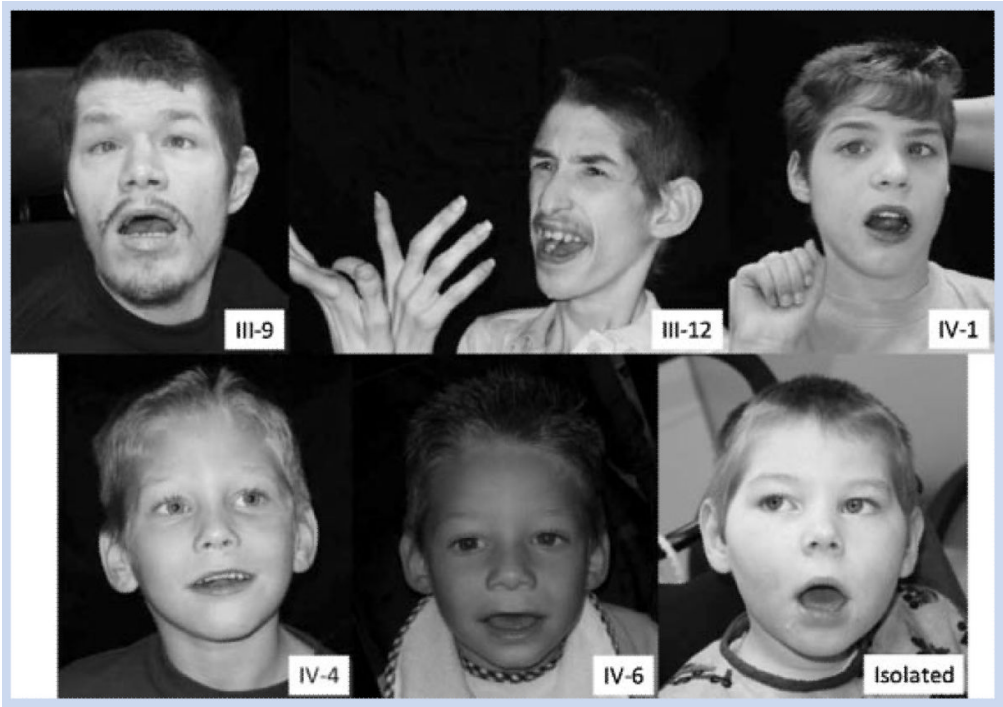

FIG. 2.

Facial appearance of five of the six affected males in Family 1 (K8310) and the single affected male in Family 2 (K9524). III-9 at age 35 years showing frontal upsweep, strabismus, open mouth and prominent jaw. III-12 at age 28 years showing microcephaly, upsweep of frontal hair, thin and gaunt face, low nasal septum, open mouth and abnormal hand posturing. IV-1 at age 15 years showing microcephaly and hypotonic face with open mouth. IV-4 at age $6^{11 / 12}$ years showing microcephaly and frontal upsweep of hair, but otherwise normal face. IV-6 at age 14 months showing microcephaly, upswept frontal hair, triangular face, wide mouth and thin upper lip. The isolated case in Family 2 is shown at age 6 years with microcephaly and hypotonic face with open mouth. 


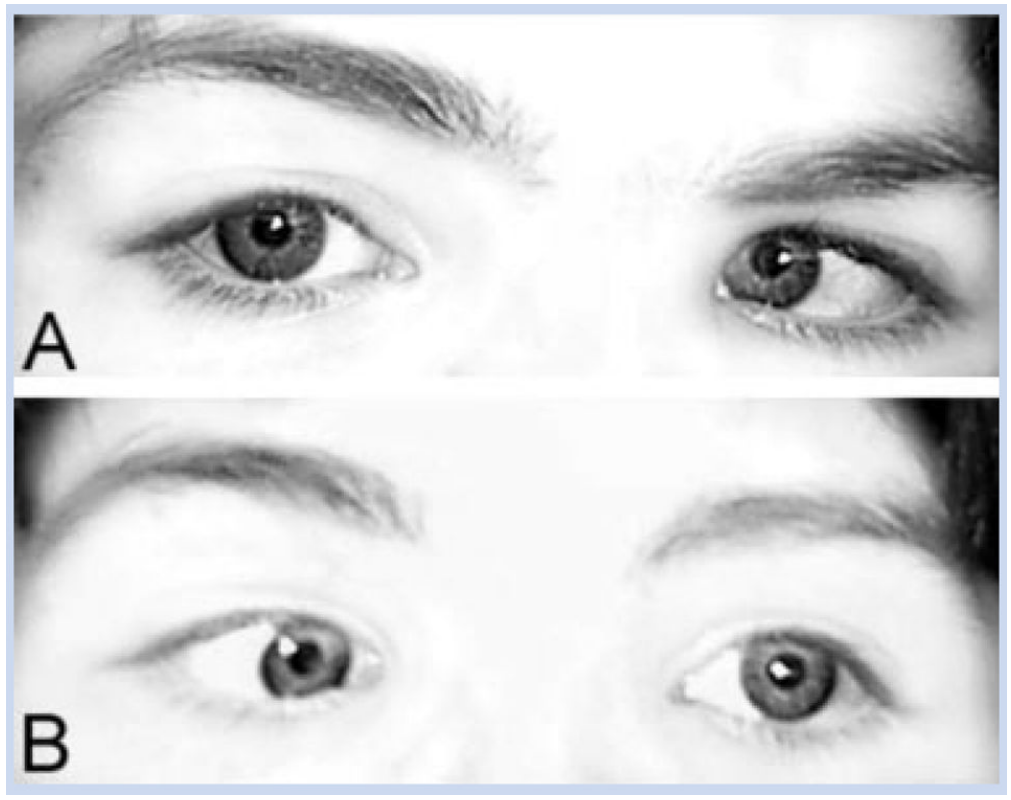

FIG. 3.

Bilateral abnormal horizontal eye movements in IV-I (family 1, K831) when looking to the right (A) and to the left (B). Although there were occasions that the eyes could almost be laterally buried, the majority of the time he exhibited a bilateral cranial nerve VI paresis on examination. 

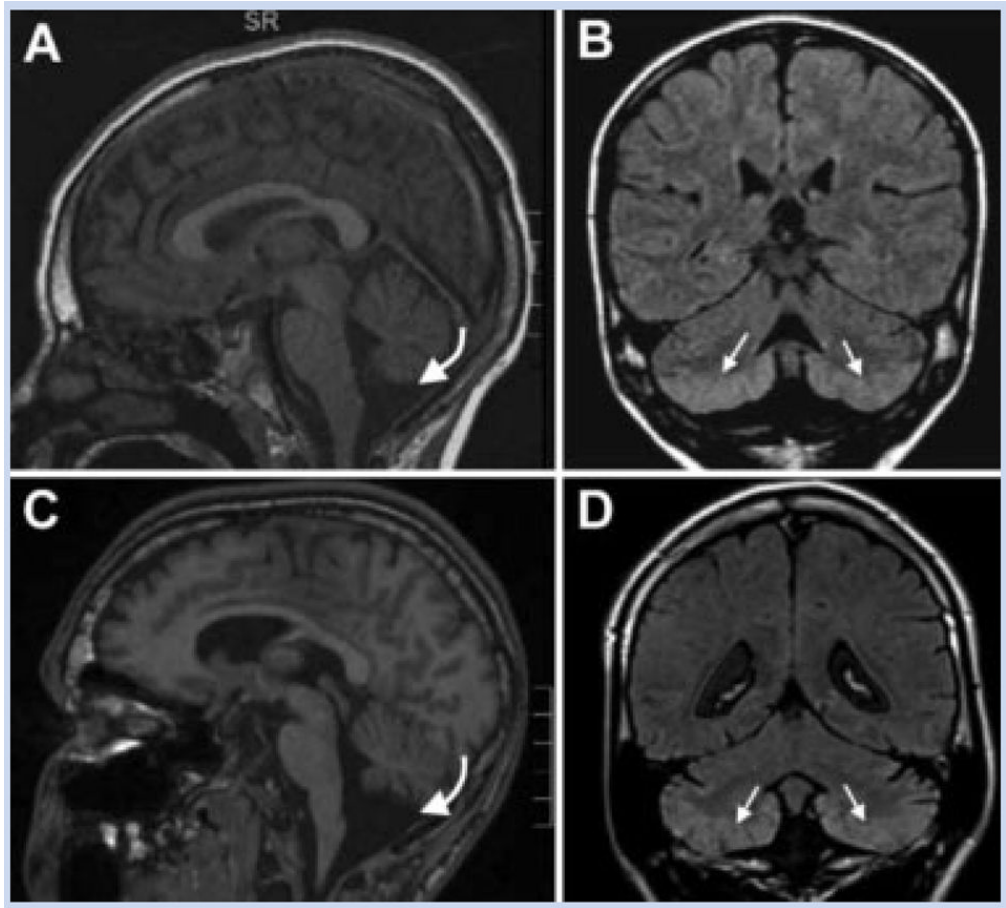

FIG. 4.

Brain magnetic resonance imaging on maternal half-brothers (IV-1 and IV-4, Family 1, K8310) using a 1.5 T magnet. A,B: Sagittal T1-weighted and coronal FLAIR images respectively on IV-4 at age 7 years. C,D: sagittal T1-weighted and coronal FLAIR images on IV-1 at age 14 years. A,C: Volume loss secondary to focal atrophy and/or hypoplasia of the inferior cerebellar vermis with prominent cerebral spinal fluid space at the cisterna magna in both patients (curved white arrows). The volume loss secondary to focal atrophy and/or hypoplasia is more conspicuous in the oldest patient, IV-1. B,D: Subtle abnormally high signal in the inferior aspect of the cerebellar hemispheres on coronal FLAIR sequences in IV-4 and IV-1 (straight white arrows) which may indicate some degree of gliosis. 

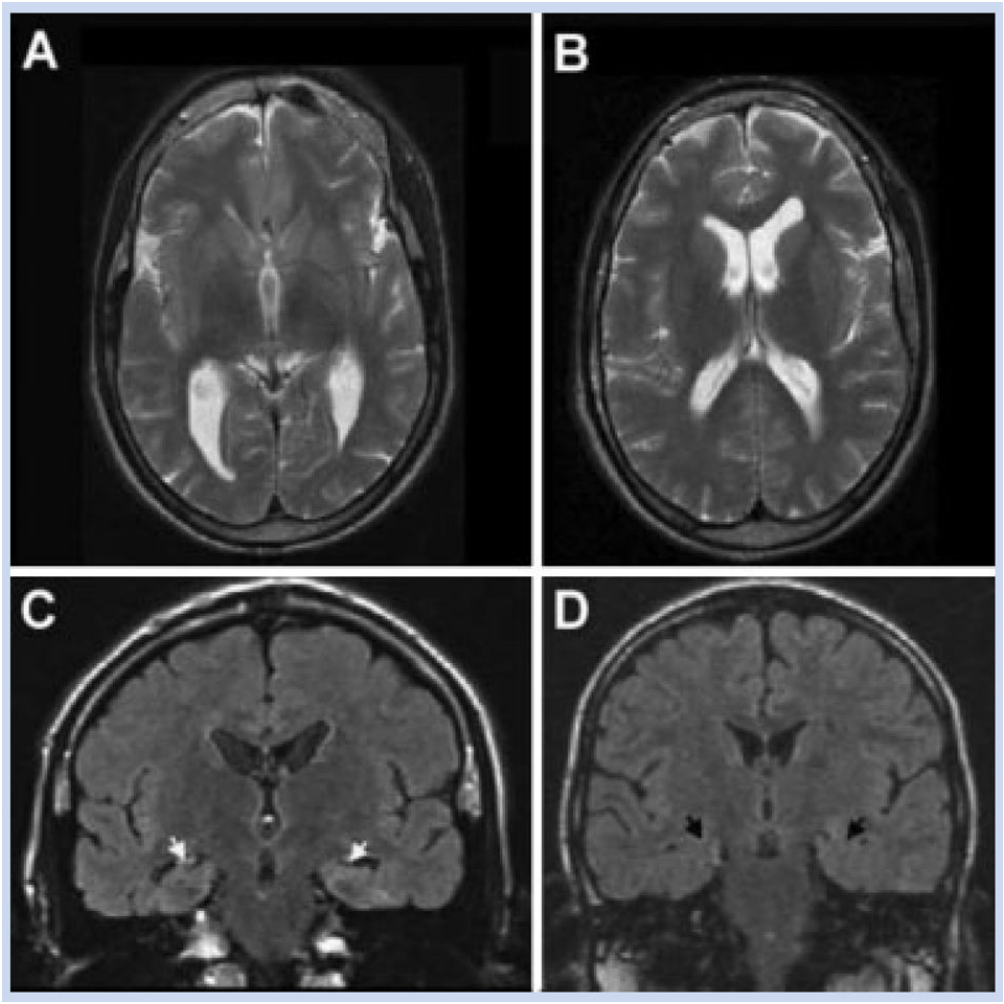

FIG. 5.

Transverse spin-echo T2-weighted magnetic resonance images of the brain. A,B: Prominent lateral ventricles in IV-1. Brain MRI coronal FLAIR (Fluid Attenuation Inversion Recover) images. C,D: Mild reduction in size and increase signal of the hippocampi (white arrows image C) in IV-1 in comparison to image D which shows normal size and signal of the hippocampi on IV-4 (black arrows). 


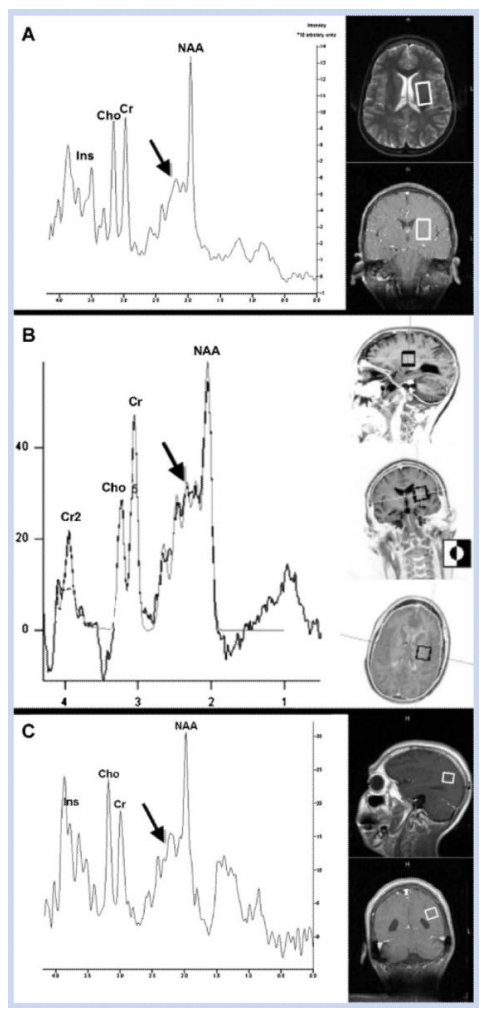

FIG. 6.

Single voxel short echo time magnetic resonance spectroscopy (MRS) performed in a $1.5 \mathrm{~T}$ magnet (TE: 25-30 ms) demonstrated a strikingly similar abnormally prominent Glutamate/ Glutamine shoulder (arrows) in all three spectra. The volumes of interest are located in the left basal ganglia in IV-4 (A), the left basal ganglia in IV-1 (B), and the left parietal white matter in IV-1 (C). The illustrations in the panels on the right indicate the position of the voxels. Standard spectral peaks are illustrated for inositol (Ins), choline (Cho), creatine (Cr), and $\mathrm{N}$-acetylaspartate (NAA). 
TABLE 1

Clinical Findings in Affected Males in Two Families With SLC9A6 Mutations

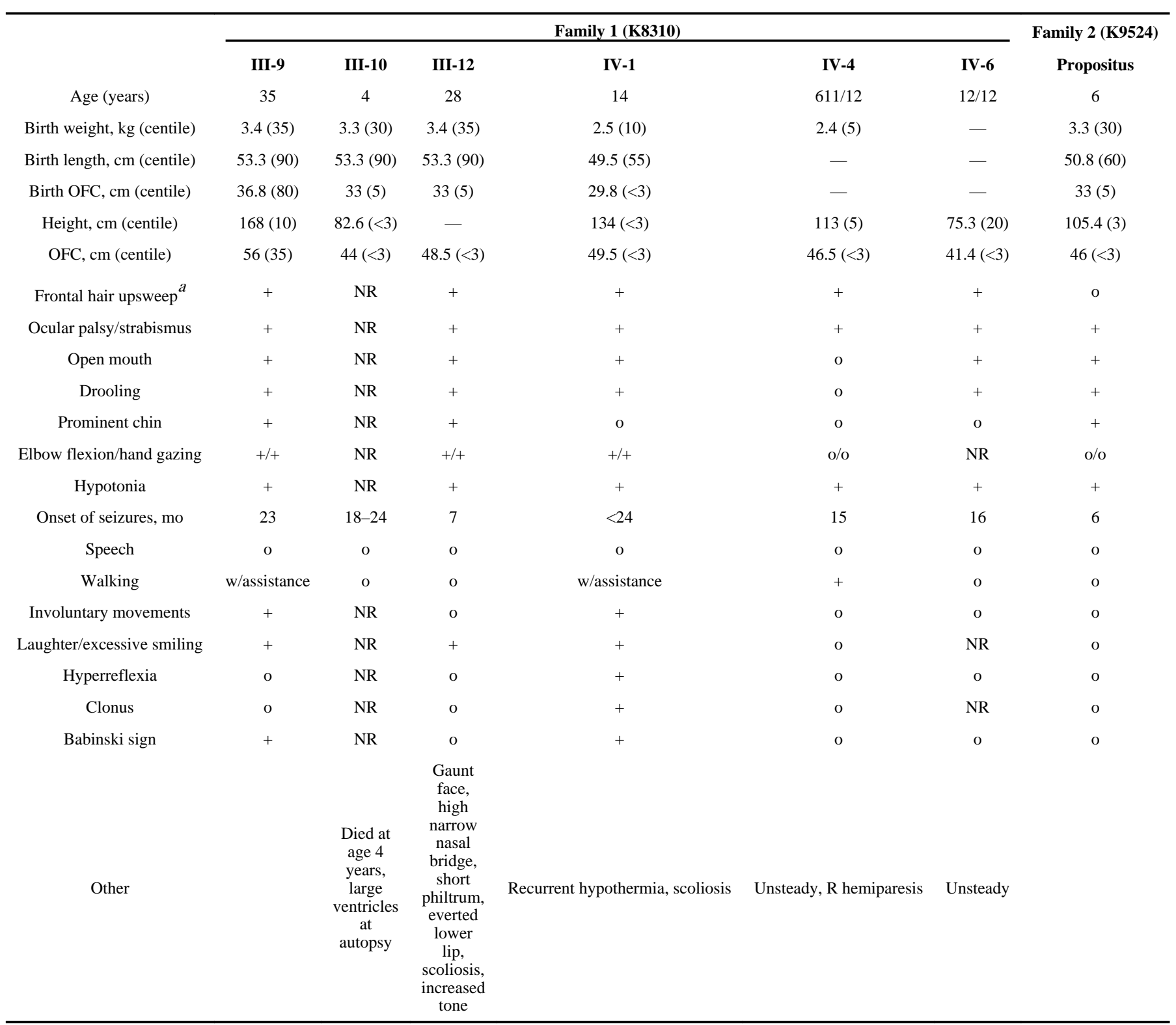

+, finding present; o, finding not present; NR, unknown or observation not recorded.

${ }^{a}$ A non-carrier female in K8310 also has frontal hair upsweep. 
TABLE II

Test Results for Stanford-Binet Intelligence Test, Fourth Edition, on Females in Family 1

\begin{tabular}{lcrccc}
\hline Family 1 K8310 & Verbal & Abstract/visual & Quantitative & Short term memory & composite \\
II-4, carrier & 49 & 70 & 62 & 40 & 49 \\
III-11, carrier & 64 & 88 & 62 & 60 & 62 \\
III-16, noncarrier & 75 & 110 & 64 & 49 & 71 \\
\hline
\end{tabular}




\section{TABLE III}

SLC9A6 Mutation Summary

\begin{tabular}{llll}
\hline Family & Mutation & Protein alteration & Report \\
Norwegian & c.764_769delAAAGTG & p.E255_S256del & Gilfillan et al. [2008] \\
Swedish & C.1402C $>$ T & p.R468X & Gilfillan et al. [2008] \\
British & c.507+1delGTAA & p.V144_R1G9del & Gilfillan et al. [2008] \\
Afrikaner & c.512_513delAT & p.H171fs & Christianson et al. [1999]; Gilfillan et al. [2008] \\
K8310 & C.1402C $>T$ & p.R468X & This report \\
K9524 & C.1219C $>$ T & p.Q407X & This report \\
\hline
\end{tabular}

